\title{
Alignment parameter calibration for IMU using the Taguchi method for image deblurring
}

\author{
${ }^{* 1}$ Kit Yan Chan, ${ }^{1}$ Nimali Rajakaruna, ${ }^{2}$ Ulrich Engelke, ${ }^{1}$ Iain Murray, and ${ }^{1}$ Nimsiri Abhayasinghe \\ ${ }^{1}$ Department of Electrical and Computer Engineering, Curtin University, Australia. \\ ${ }^{2}$ Digital Productivity Flagship of the Commonwealth Scientific and Industrial Research Organisation \\ (CSIRO), Australia. \\ *Kit Yan Chan is the corresponding author. e-mail: kit.chan@curtin.edu.au
}

Abstract - Inertial measurement units (IMUs) utilized in smartphones can be used to detect camera motion during exposure, in order to improve image quality degraded with blur through long hand-held exposure. Based on the captured camera motion, blur in images can be removed when an appropriate deblurring filter is used. However, two research issues have not been addressed: (a) the calibration of alignment parameters for the IMU has not been addressed. When inappropriate alignment parameters are used for the IMU, the camera motion would not be captured accurately and the deblurring effectiveness can be downgraded. (b) Also selection of an appropriate deblurring filter correlated with the image quality has still not been addressed. Without the use of an appropriate deblurring filter, the image quality could not be optimal. This paper proposes a systematic method, namely the Taguchi method, which is a robust and systematic approach for designing reliable and high-precision devices, in order to perform the alignment parameter calibration for the IMU and filter selection. The Taguchi method conducts a small number of systematic experiments based on orthogonal arrays. It studies the impact of the alignment parameters and appropriate deblurring filter, which attempts to perform an effective deblurring. Several widely adopted image quality metrics are used to evaluate the deblurred images generated by the proposed Taguchi method. Experimental results show that the quality of deblurred images achieved by the proposed Taguchi method is better than those obtained by deblurring methods which are not involved with the alignment 
parameter calibration and filter selection. Also, much less computational effort is required by the Taguchi method when comparing with the commonly used optimization methods for determining alignment parameters and deblurring filter.

Index terms: Inertial measurement unit (IMU), Taguchi method, image deblurring, orthogonal array, alignment parameters

\section{INTRODUCTION}

The use of smartphones to capture digital photographs is becoming increasingly popular. Based on the financial report issued by Gartner [9], 968 million smartphones were sold worldwide in 2013, compared to 680 million Smart Phones for a year ago. Every day, numerous digital photographs are captured by Smart Phones, and those digital photographs are being posted daily on online social networks such as Facebook and twitter. We want the images to be a faithful representation of the scene that we wish to capture. However, these images are never perfect because image blurring can be caused by camera motion through long hand-held exposure. Depending on the camera, this can be one of the prime causes of poor image quality in image acquisition and can significantly degrade the structure of sharp images.

Deblurring is usually performed offline using mathematical algorithms in order to improve image quality. Deblurring algorithms [30,44] use two captured images, the correlation between which is used for determining the deblurring kernel. More recently, Fergus et al. [8] developed a method using a single image for handling slight blurring such as camera tremble. It attempts to determine the camera motion based on the initial blur kernel, which is estimated by the heuristic information of camera motion. Another deblurring method was proposed by Shan et al. [35] based on a unified probabilistic model consisting of both blur kernel estimation and deblurred image restoration. However, they have the common limitations that they work only under specified blur patterns. Also, they have high computational costs and are therefore not suitable for implementing on real-time systems, which have limited computational power. Although recent blind deblurring algorithms have been developed with good results on evaluating with a huge number of images 
$[42,43]$, camera motion information during image exposure which is available on smartphones have not been used on the deblurring.

Manufacturers are desperate to find ways to improve the image quality by removing image blur. Thanks to the advanced sensor technologies, inertial measurement units (IMUs) [15] have commonly been used in modern smartphones and tablets to capture the motion of the camera path [19], as they are small in size, inexpensive, reliable, and have low power consumption. They can be used to capture the camera motion precisely. Based on the captured motion information, navigation [20], orientation determination [4], and human motion tracking [47] can be implemented. Also, deblurring can be performed based on the captured camera motion, in order to remove image blur caused by camera motion [15]. Hence, image quality can be improved.

In spite of the smaller size and cost effectiveness of IMU, the error behaviour of IMU must be appropriately treated in order to turn the raw sensor measurements into reliable and useful data for motion tracking [40], as both deterministic and stochastic errors exist in IMU. These errors affect the precision on capturing the camera motion which is the key to perform an effective deblurring. The deterministic error is caused by zero-offset bias, scale factor and misalignment; the stochastic error is caused by random noise of the IMU. Calibration approaches have been developed by tuning the inbuilt parameters of the IMU in order to reduce those errors [40]. However, the IMU is often purchased commercially and has been implemented in the Smartphone for capturing camera motion, making changes to any inbuilt parameters in the IMU impractical. Hence, it is impractical to open the IMU to address the zero-offset bias, scale factor, and random noise. As the camera moves, the alignment parameters correlating the origins of the camera and the frame of interest must be calibrated in order to achieve good image quality. It is necessary to align the motion trajectory with the captured source of interest. Appropriate deblurring can be done only when the alignment parameters are correctly calibrated. These alignment parameters are more practical to be tuned than tuning the inbuilt parameters, as the alignment parameters can be tuned without opening the IMU implemented in the smartphone. 
However, recent research has not addressed misalignment between the camera and the captured source of interest, although Sindelar and Sroubek [36] have implemented the IMU on the smartphone for real-time deblurring. Also, Bae et al. [3], Horstmeyer [12] and Sindelar and Sroubek [36] assumed that there is no misalignment between the camera and the frame of interest. The deblurring is developed with the assumption that no misalignment exists. Although Joshi et al. [15] developed a drift compensator in order to reduce drift caused by the randomly noisy measure present in the sensor reading, misalignment is still not addressed. The deblurring effectiveness can be downgraded, when inappropriate alignment parameters are used. Apart from not addressing misalignment, this research has used only a state-of-art filter for deblurring images. Although there has been a considerable amount of work on filter design, selection of appropriate filters is essential as the effectiveness of deblurring is correlated with the filter. Without the appropriate filter, the effectiveness of deblurring cannot be optimized.

In this paper, a systematic method, namely the Taguchi method, which is commonly used for the design of reliable and high-precision devices [38], is proposed for calibrating alignment parameters and selecting the appropriate filter, in order to perform effective image deblurring. In quality optimization, the Taguchi method has been successfully used to design reliable processes and high-quality products at low cost for various items $[2,7,11,29,34,37]$. Here, we consider the deblurring design to be the design of a high quality product [22]. Similar to the design of high quality products, which aim to produce the functionality by approximating the ideal function as closely as possible, the effective deblurring mechanism design aims to process blurred images so that they are faithful representations of the original scene. In accordance with the Taguchi method, the alignment parameters and deblurring filter are calibrated and selected in an inner orthogonal array. The Taguchi method conducts a small but systematic number of experiments based on orthogonal arrays to study the impact of each alignment parameter and the deblurring filter. It attempts to determine the alignment parameter setting and the filter in order to achieve effective image deblurring.

The calibrated results obtained by the Taguchi method were evaluated through two phases. First, the results were compared with several commonly-used deblurring methods, where the alignment parameters defaulted by the camera manufacturer were engaged with those deblurring methods. Several commonly used 
image quality metrics [41] were used to evaluate whether significantly better results can be obtained by the calibrated parameters and the defaulted parameters. Second, several commonly-used optimization methods namely, genetic algorithm [10], simulated annealing algorithm [16] and particle swarm optimization algorithm [28] were used to determine the alignment parameters and the deblurring filter. These optimization methods were used, as they are effective for solving difficult optimization problems without gradient information which is the case of this deblurring problem. We attempt to evaluate whether the Taguchi method can obtain better results than those optimization methods, when similar computational time is used. The results show that the calibrated parameters can achieve a more effective deblurring than the defaulted parameters. Also the experimental results show that the image quality achieved by the proposed Taguchi method is better than those obtained by the commonly used optimization methods, and much less computational effort is required by the proposed method.

The remainder of the paper is organized as follows. Section 2 provides a brief description of using the Taguchi method for quality optimization. Section 3 defines and describes the formulation and mechanisms for image deblurring design. In Section 4, the main operations of the Taguchi method for image deblurring design, which involve the parameter calibration and filter selection, are discussed. In Section 5, comparison results with the defaulted parameters and the commonly-used optimization methods are presented. Finally, a conclusion, regarding the results obtained by the Taguchi method and the advantages of the Taguchi method, are presented in Section 5.

\section{QUALITY OPTIMIZATION USING TAGUCHI METHOD}

Before developing the Taguchi method for calibrating the IMU, this section briefly describes the Taguchi method which has been widely used for quality optimization of industrial systems [11,37]. The first step of quality optimization is to initiate the basic functional prototype design based on the knowledge of the industrial system. It attempts to configure the system attributes undergoing analysis. As the quality of the initial design is usually far from the optimal design quality, it is necessary to determine an appropriate set of design factors in order to maximize the system quality. 
The appropriate set of design factors can be established by means of a "full factorial" design, where all possible levels of all design factors are considered. When a system has $n$ design factors and each of them has $k$ levels, the total number of combinations for the "full factorial" approach is $k^{n}$. When the number of design factors is large, it is almost impossible to test all possible permutations for all design factors. For example, given an industrial system with 10 design factors with each design factor having three levels, a full factorial approach requires examining 59049 (i.e. $3^{10}$ ) experiments. Alternatively, the trial and error method can be used by trialing the design factors with different levels one at a time until a reasonable design with certain quality is found. However, numerous experiments are required in order to arrive at a reasonable design. To keep the number of experiments low, the Taguchi method is commonly used for quality optimization [11, 37]. It studies the effect of design factors simultaneously by planning matrix experiments using an orthogonal array, which studies a design factor domain with the smallest number of experiments [38]. Based on the orthogonal array, the domain of the design factors can be studied systematically and efficiently.

As an example, we consider an epoxy dispensing system with four design factors which include three operational parameters and 1 component parameter [17]. The three operational parameters are the values of the injection pressure (ranging from 1 to 4 psi), the motor speed (ranging from 400 to 1000 rotations per minute), and the distance between the nozzle and the dispensing target (ranging from 500 to 1500 steps), which represent the first three Design Factors A, B and C respectively. Here, three levels with respect to the ranges of the operational parameters are considered. The component parameter includes three kinds of epoxy material namely epoxy I, epoxy II and epoxy III; these three kinds of epoxy material correspond to the three levels. Epoxy I, epoxy II and epoxy III are with respect to level 1, level 2 and level 3 for the Design Factor D respectively. Table I illustrates the three operational parameters (i.e. Design Factors A, B and C) with respect to the three levels and it also illustrates the component parameter (i.e. Design Factor D) with respect to the three levels. We attempt to control the epoxy size of the epoxy dispensing system by determining the appropriate setting of the four Design Factors. 
Table 1 Design Factors for the epoxy dispensing system.

\begin{tabular}{|c|c|c|c|c|}
\hline \multirow{2}{*}{} & \multicolumn{3}{|c|}{ Operational parameters } & Component \\
& \multicolumn{2}{|c|}{ parameter } \\
\cline { 2 - 5 } & Design Factor A & Design Factor B & Design Factor C & Design Factor D \\
& (injection pressure) & (motor speed) & (distance between & (epoxy material) \\
& & & nozzle and dispensing & \\
\hline Level 1 & $1 \mathrm{psi}$ & $400 \mathrm{r} / \mathrm{min}$ & 500 steps & Epoxy I \\
\hline Level 2 & $2.5 \mathrm{psi}$ & $700 \mathrm{r} / \mathrm{min}$ & 1000 steps & Epoxy II \\
\hline Level 3 & $4 \mathrm{psi}$ & $1000 \mathrm{r} / \mathrm{min}$ & 1500 steps & Epoxy III \\
\hline
\end{tabular}

For the epoxy dispensing system with four Design Factors with each Design Factor having three levels, a full factorial design with 81 (i.e.: $3^{4}$ ) experiments is required. However, only nine experiments are required, when an orthogonal array, $L_{9}\left(3^{4}\right)$ (shown in Table 2), is used. In $L_{9}\left(3^{4}\right)$, there are four columns representing the Design Factors A, B, C and D, each of which has three levels. The number of rows represents the system configurations to be tested with respect to the experimental level defined by the row, and the number of columns represents the number of design factors which are studied. Compared with the full factorial design, 72 (i.e. 81-9) experiments are saved when the orthogonal array, $L_{9}\left(3^{4}\right)$ is used. In this design problem, assuming that approximately two hours are required for each experiment, 162 hours are required for full factorial design to be conducted. Hereby, only 18 hours are required when the orthogonal array, $L_{9}\left(3^{4}\right)$, is used. Comparing this with the full factorial design, 144 hours can be saved in this design problem. Therefore, a significant amount of experimental time can be saved when $L_{9}\left(3^{4}\right)$ is used. Also, the nine experiments in $L_{9}\left(3^{4}\right)$ have the pairwise balancing property, whereby every experiment of a Design Factor is conducted the same number of times. Hence, the experimental levels defined by the columns are mutually orthogonal. It 
minimizes the number of required experiments, while retaining the pairwise balancing property. Only nine experiments are sufficient to evaluate the main effect of each Design Factor in order to determine the appropriate setting of the epoxy dispensing system.

Table 2 Orthogonal array $\left(L_{9}\left(3^{4}\right)\right)$.

\begin{tabular}{|c|c|c|c|c|}
\hline Experiments & Design Factor A & Design Factor B & Design Factor C & Design Factor D \\
\hline $\mathbf{1}$ & Level 1 & Level 1 & Level 1 & Level 1 \\
\hline $\mathbf{2}$ & Level 1 & Level 2 & Level 2 & Level 2 \\
\hline $\mathbf{3}$ & Level 1 & Level 3 & Level 3 & Level 3 \\
\hline $\mathbf{4}$ & Level 2 & Level 1 & Level 2 & Level 3 \\
\hline $\mathbf{5}$ & Level 2 & Level 2 & Level 3 & Level 1 \\
\hline $\mathbf{6}$ & Level 2 & Level 3 & Level 1 & Level 2 \\
\hline $\mathbf{7}$ & Level 3 & Level 1 & Level 3 & Level 2 \\
\hline $\mathbf{8}$ & Level 3 & Level 2 & Level 1 & Level 3 \\
\hline $\mathbf{9}$ & Level 3 & Level 3 & Level 2 & Level 1 \\
\hline
\end{tabular}

\section{IMU FOR IMAGE DEBLURRING}

Two steps are involved in deblurring images using the camera motion captured by the IMU. First, the IMU is used to measuring a sequence of angular and linear velocities for the camera motion when capturing the image. Second, a blur kernel is developed using the captured data of the camera motion. It is determined with respect to the image quality by tuning the alignment parameters in order to improve the deblurring effectiveness.

\subsection{Capture of camera motion}

Figure 1 shows the scene plane of the image captured by the camera. It illustrates the alignment parameters, $\bar{\phi}=(a, b, l)$, which consists of the coordinate of the aligned focus, namely $a$ and $b$, and the aligned distance 
between the scene plane and the camera, namely $l$. The angular and the linear velocities of the camera motion are captured by the IMU when the image is being captured.

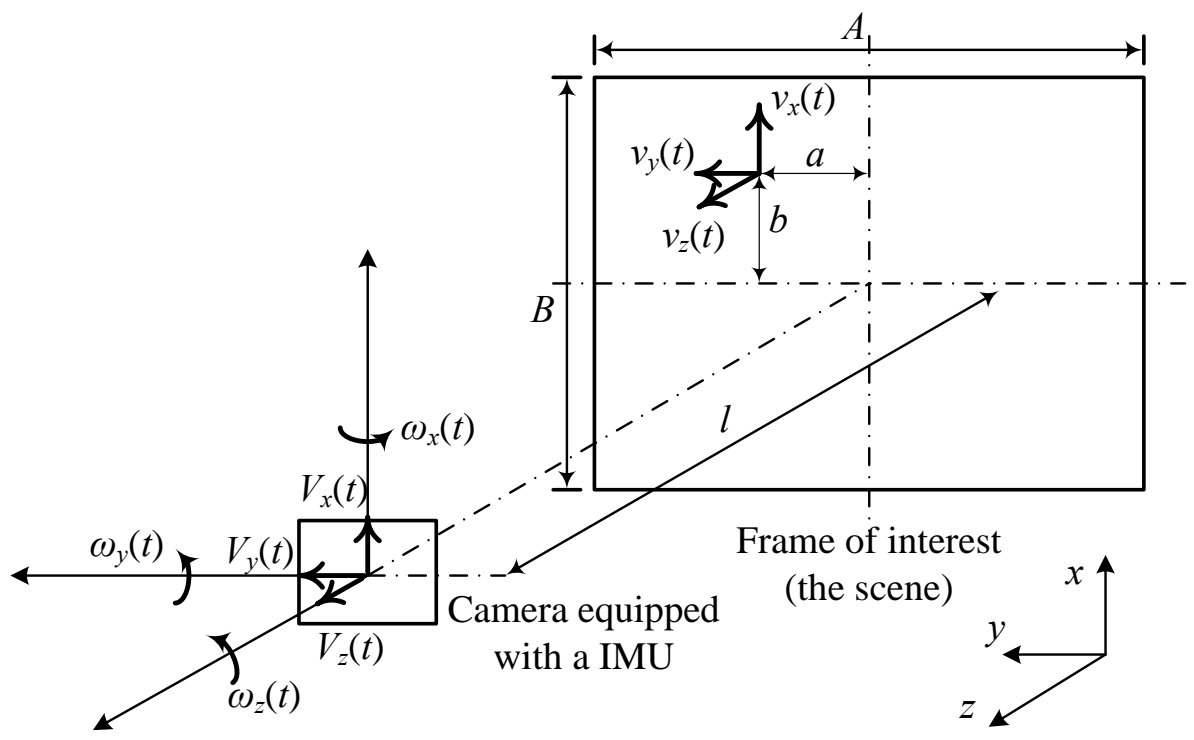

Figure 1 Illustration of the alignment parameters.

The angular velocities captured by the IMU are denoted by $\omega_{x}(t), \omega_{y}(t)$, and $\omega_{z}(t)$, with respect to the $x, y$, and $z$ axes respectively, where $t$ is the caption time with $t=0, T_{s}, 2 T_{s}, \ldots, N_{\mathrm{s}} \cdot T_{s} ; T_{s}$ is the sampling period; and $N_{\mathrm{s}}$ samples of angular velocities are captured. The linear velocities captured by the IMU are denoted by $V_{x}(t), V_{y}(t)$, and $V_{z}(t)$, with respect to the $x, y$, and $z$ axes respectively. The linear velocities at the alignment points, namely $v_{x}(t), v_{y}(t)$, and $v_{z}(t)$ are determined based on (1a), (1b) and (1c) with respect to the $x, y, z$ axes respectively as,

$$
\begin{aligned}
& v_{x}(t)=-V_{x}(t)+l \omega_{y}(t)+a \omega_{z}(t), \\
& v_{y}(t)=-V_{y}(t)-l \cdot \omega_{x}(t)-b \cdot \omega_{z}(t), \\
& v_{z}(t)=-V_{z}(t)-a \cdot \omega_{x}(t)+b \cdot \omega_{z}(t) .
\end{aligned}
$$


As people usually stand on taking photos, the linear velocities, $V_{x}(t), V_{y}(t)$, and $V_{z}(t)$, are generally small. The camera motion is mostly caused from hand shake. Hence, the angular velocities, $\omega_{x}(t), \omega_{y}(t)$, and $\omega_{z}(t)$, usually exist. Also based on the motion data captured by the IMU, it confirmed that the linear velocities are generally near zero and the angular velocities usually exist. As $V_{x}(t), V_{y}(t)$, and $V_{z}(t)$, are very small compared to the $\omega_{x}(t), \omega_{y}(t)$, and $\omega_{z}(t)$ with respect to the velocity of the point on the scene, $V_{x}(t)$, $V_{y}(t)$, and $V_{z}(t)$ can be omitted from (1a), (1b) and (1c). Hence, $v_{x}(t), v_{y}(t)$, and $v_{z}(t)$ can be computed based on (2a), (2b) and (2c) respectively as:

$$
\begin{aligned}
& v_{x}(t)=l \cdot \omega_{y}(t)+a \cdot \omega_{z}(t), \\
& v_{y}(t)=-l \cdot \omega_{x}(t)-b \cdot \omega_{z}(t), \\
& v_{z}(t)=-a \cdot \omega_{x}(t)+b \cdot \omega_{z}(t) .
\end{aligned}
$$

The displacement of the motion blur can be determined by iterating the three linear velocity components, $v_{x}(t), v_{y}(t)$, and $v_{z}(t)$, given in (2a), (2b) and (2c) using the trapezoid rule. Hence, determination of the displacement of the motion blur, namely $D_{x}(t), D_{y}(t)$ and $D_{z}(t)$, with respect to $x, y$ and $z$, is given as:

$$
\begin{aligned}
& D_{x}(t)=D_{x}\left(t-T_{s}\right)+\left(v_{x}(t)-v_{x}(t-1)\right) \cdot T_{s}, \\
& D_{y}(t)=D_{y}\left(t-T_{s}\right)+\left(v_{y}(t)-v_{y}(t-1)\right) \cdot T_{s}, \\
& D_{z}(t)=D_{z}\left(t-T_{s}\right)+\left(v_{z}(t)-v_{z}(t-1)\right) \cdot T_{s} .
\end{aligned}
$$

\subsection{Tuning of the alignment parameters}

Based on the mechanism developed by Jiang et al. [14], the blur kernel can be determined using the energy level of each pixel of the motion blur. The blur kernel, $\bar{\tau}$, is determined with respect to the motion blur given in (3a), (3b) and (3c), where the dimensions of $\bar{\tau}$ with the matrix size of $n_{x} \times n_{y}$ are given as 
$n_{x}=\frac{\max _{i=1,2, \ldots, N_{s}}\left(D_{x}\left(i \cdot T_{s}\right)\right)}{p_{s}}$

and $n_{y}=\frac{\max _{i=1,2, \ldots, N_{s}}\left(D_{y}\left(i \cdot T_{s}\right)\right)}{p_{s}}$;

$p_{s}$ is the pixel size of the image; and each element in $\bar{\tau}$ is denoted as $\tau_{i, j}$ with $i=1,2, . ., n_{x}^{\Gamma}$ and $j=1,2, \ldots, n_{y}^{\Gamma}$ and $\tau_{i, j}$ is given by,

$$
\tau_{i, j}=\frac{\sum_{k=1}^{N_{s}} F_{i, j}(k)}{\sum_{i=1}^{n_{x}} \sum_{j=1}^{n_{y}} \sum_{k=1}^{N_{s}} F_{i, j}(k)},
$$

with $F_{i, j}(k)=\left\{\begin{array}{l}1, \text { if } i=\operatorname{round}\left(\frac{D_{x}\left(k \cdot T_{s}\right)}{p_{s}}\right) \text { and } j=\operatorname{round}\left(\frac{D_{y}\left(k \cdot T_{s}\right)}{p_{s}}\right) \\ 0, \text { otherwise. }\end{array}\right.$

$\tau_{i, j}$ is correlated with the displacement of the motion blur given in (3a), (3b) and (3c), and the displacement of the motion blur is correlated with the alignment parameters $\bar{\phi}=(a, b, l)$ and the captured angular velocities $\bar{\omega}(t)=\left(\omega_{x}(t), \omega_{y}(t), \omega_{z}(t)\right)$ with $t=0, T_{s}, 2 T_{s}, \ldots, N_{s} \cdot T_{s}$. Hence, a correlation function, namely $\Phi_{\tau}$, is defined in order to correlate the blur kernel, $\bar{\tau}$, with the alignment parameters $\bar{\phi}$ and the sequence of captured angular velocities $\bar{\omega}$, where $\bar{\omega}=\left(\bar{\omega}(0), \bar{\omega}\left(T_{s}\right), \bar{\omega}\left(2 \cdot T_{s}\right), \ldots \bar{\omega}\left(N_{s} \cdot T_{s}\right)\right) . \Phi_{\tau}$ is given as:

$$
\bar{\tau}=\Phi_{\tau}(\bar{\omega}, \bar{\phi})
$$

When a blurred image, $\bar{I}^{b}$, is captured by the camera, a deblurring filter, namely $\Phi_{F}$, engaged with $\bar{\tau}$ can be used to generate an enhanced image, $\bar{I}^{e}$, which is given as:

$$
\bar{I}^{e}=\Phi_{F}\left(\bar{I}^{b}, \bar{\tau}\right)=\Phi_{F}\left(\bar{I}^{b}, \Phi_{\tau}(\bar{\omega}, \bar{\phi})\right)
$$


After $\bar{I}^{e}$ has been generated, a specified image quality measure, namely, $J(\bar{\phi})$, given in (8), can be evaluated based on a perceptual image quality model, $\Phi_{P}$, which is ideally independent of the content of the visual scene. As the angular velocity, $\bar{\omega}$, for the camera motion is captured by the IMU, it cannot be adjusted. Also, the blurred image, $\bar{I}^{b}$, cannot be changed as this is captured by the camera. We can only adjust the alignment parameters, $\bar{\phi}$, in order to fine-tune the image quality for the enhanced image. The alignment parameters, $\bar{\phi}$, can be fine-tuned with respect to $J(\bar{\phi})$ as:

$$
J(\bar{\phi})=\Phi_{P}\left(\Phi_{F}\left(\bar{I}^{b}, \Phi_{\tau}(\bar{\omega}, \bar{\phi})\right)\right)
$$

Assume that the image quality is better when $J(\bar{\phi})$ is smaller. Determination of the appropriate $\bar{\phi}$ and $\Phi_{F}$ is the minimization problem. When the image quality with a larger $J(\bar{\phi})$ is better, determination of the appropriate $\bar{\phi}$ and $\Phi_{F}$ is a maximization problem. For example, the image quality regarding the degree of blur is the-smaller-the-better performance characteristic [21]. The image quality regarding the gradients and edges of the objects in the image is the-larger-the-better performance characteristic [33]. Based on the formulation of (8), different image quality measures, $\Phi_{P}$, can be used in order to evaluate the deblurring performance and also the deblurring performance can be optimized with respect to different deblurring filters, $\Phi_{F}$. It overcomes the limitation of the formulation developed in [12] where only a single deblurring filter and a single image quality measure are used to develop the blur kernel.

Solving $J(\bar{\phi})$ is difficult, as the deblurring filter, $\Phi_{F}$, and perceptual image quality model, $\Phi_{P}$, are generally nonlinear. Also, the computational cost of evaluating $J(\bar{\phi})$ is high, as it needs to compute through the three functions, $\Phi_{\tau}, \Phi_{F}$, to $\Phi_{P}$. In Section IV, the Taguchi method is proposed to determine an appropriate $\bar{\phi}$ and the deblurring filter, $\Phi_{F}$, by conducting systematic trials. It attempts to determine an appropriate $\bar{\phi}$ and $\Phi_{F}$ with little computational effort. 


\section{TAGUCHI METHOD FOR IMU CALIBRATION}

This section demonstrates how the Taguchi method is used to calibrate the IMU for deblurring images. The Taguchi method can be divided into two main tasks. Firstly, the design factors of the IMU are quantized into certain levels and the orthogonal array is selected with respect to the number of design factors and the quantized levels. Based on the combinations of the selected orthogonal array, the experiments are conducted with respect to the design factors. Secondly, the main effects of each design factor are analyzed based on the experimental results. The most appropriate levels for each design factor are determined based on the level with the best main effect. Hence, the design factors of the IMU can be recommended.

\subsection{Orthogonal array and systematic experiments}

In this research, the Sony Xperia TX smartphone equipped with a camera and an IMU was used to capture the blurred image and the angular velocities associated with the camera motion respectively, where the sampling time of capturing the angular velocities was $5 \mathrm{~ms}$ and 14 samples of angular velocities are captured. We consider three alignment parameters of the $\operatorname{IMU}(a, b, l)$ as the first three Design Factors of the Taguchi Method, where the alignment ranges are provided by the IMU manufacturer i.e., $a \in[-1.5 . .1 .5]$, $b \in[-1.5 . .1 .5]$ and $l \in[3.1 . .4 .9]$. The three state-of-art deblurring filters [13] namely Wiener filter, Lucy-Richardon filter and regularized filter, are considered as the fourth Design Factor, where the regularized filter [13] is an enhanced version of the Wiener filter.

The number of levels of the design factors in the Taguchi method affects the efficiency of the calibration. For calibrating a system with only linear design factors, two levels are used since two points define a line. For calibrating a system involving quadratic design factors, three levels are used since the two extremes and the midpoint can represent the quadratic nature. For calibrating a system involving cubic design factors, four levels are used since two extremes and two points in between can represent the cubic nature. Extra levels allow for the examination of complicated nonlinear systems, but more experimental time is required. As the number of design factors that need to be calibrated is not high, we start to use a small 
orthogonal array namely, $L_{9}\left(3^{4}\right)$, which involves four design factors with each design factor having three levels. We divide the three alignment parameters of the IMU $(a, b, l)$ into three levels as shown in Table 3, with each of the three levels being divided equally with respect to the alignment ranges i.e., $a \in[-1.5 . .1 .5]$, $b \in[-1.5 . .1 .5]$ and $l \in[3.1 . .4 .9]$. As an instance, Levels 1,2 and 3 for parameter $a$ are with respect to -1.5 , 0.0 and 1.5 respectively. For the filter, Levels 1,2 and 3 are with respect to the Wiener filter, Lucy-Richardon filter and regularized filter respectively. Each experiment involves one of the three filters and a particular set of alignment parameters.

Table 3 The three levels for the alignment parameters and the filters.

\begin{tabular}{|l|c|c|c|c|}
\hline \multirow{2}{*}{} & \multicolumn{2}{|c|}{ Alignment parameters } & \multirow{2}{*}{ Filter } \\
\cline { 2 - 5 } & $a$ & $b$ & $l$ & \\
\hline Level 1 & -1.5 & -1.5 & 3.1 & Wiener \\
\hline Level 2 & 0.0 & 0.0 & 4 & Lucy-Richardson (L-R) \\
\hline Level 3 & 1.5 & 1.5 & 4.9 & Regularized \\
\hline
\end{tabular}

Based on the configurations of the orthogonal array, $L_{9}\left(3^{4}\right)$, the first five columns in Table 4 are used to study the impact of each alignment parameter and the filter. Based on $L_{9}\left(3^{4}\right)$, only nine experiments need to be conducted while full factorial design requires 81 (i.e. $3^{4}$ ) experiments of which there are four parameters with each parameter having three levels. Hence, 72 experiments can be saved. For the $1^{\text {st }}$ experiment configured in $L_{9}\left(3^{4}\right)$, the alignment parameters, $a, b$, and $l$, with Level 1 (i.e. $a=0.1, b=0.1$ and $l=3.1$ ) and the filter in Level 1 (i.e. Wiener filter) were used for the calibration and the image quality of the deblurred image was evaluated. For the $2^{\text {nd }}$ experiment configured in $L_{9}\left(3^{4}\right)$, $a$ with level $1, b$ with level 2 , and $l$ with level 2 , (i.e. $a=0.1, b=1.5$ and $l=4$ ) and the filter in level 2 (i.e. Lucy-Richardson filter) were used. 
Table 4 The three levels for the alignment parameters.

\begin{tabular}{|c|c|c|c|c|c|}
\hline \multirow{2}{*}{ Trials } & \multicolumn{2}{|c|}{ Alignment parameters } & \multirow{2}{*}{ Filter } & Image quality scores \\
\cline { 2 - 4 } & $a$ & $b$ & $l$ & & \\
\hline $1^{\text {st }}$ & -1.5 & -1.5 & 3.1 & Wiener & 17.634 \\
\hline $2^{\text {nd }}$ & -1.5 & 0.0 & 4 & Lucy-Richardson & 16.218 \\
\hline $3^{\text {rd }}$ & -1.5 & 1.5 & 4.9 & Regularized & 15.295 \\
\hline $4^{\text {th }}$ & 0.0 & -1.5 & 4 & Regularized & 17.080 \\
\hline $5^{\text {th }}$ & 0.0 & 0.0 & 4.9 & Wiener & 16.341 \\
\hline $6^{\text {th }}$ & 0.0 & 1.5 & 3.1 & Lucy-Richardson & 16.836 \\
\hline $7^{\text {th }}$ & 1.5 & -1.5 & 4.9 & Lucy-Richardson & 17.616 \\
\hline $8^{\text {th }}$ & 1.5 & 0.0 & 3.1 & Regularized & 17.633 \\
\hline $9^{\text {th }}$ & 1.5 & 1.5 & 4 & Wiener & 16.931 \\
\hline
\end{tabular}

In this research, an image quality analyzer recently developed by Mittal et al. [23] is used to determine the image quality denoted by $\Phi_{P}$ in (8), as it can assess image quality without prior knowledge of distortions. This approach is developed based on a collection of statistical features from a corpus of natural and undistorted images. This approach overcomes the limitation of the existing methods which either require learning from human judgments of image quality from human-rated distorted images [24, 32] or require knowledge of expected image distortions [42]. Also, this approach delivers performance comparable to several commonly-used image quality models which require training on large databases of human opinions of distorted images. Due to its reference free nature, this approach does not require a pristine, undistorted image, which would not be available in our application. Therefore, we use this approach to evaluate the quality of the deblurred image. 
Here, the computation for the nine experiments configured in $L_{9}\left(3^{4}\right)$ were conducted using Matlab 7.7 in a PC which has a CPU of Intel(R) Core(TM)2 Duo 2.66GHz and a memory of 8GB. The image quality scores with respect to the settings of the alignment parameters and the filters are shown in the fifth column of Table 4 respectively.

\subsection{Determination of design factors}

After the image quality scores of the nine experiments have been evaluated, the main effect of each design factor (i.e. the three alignment parameter and the filter type) can be separated, since the combinations of the alignment parameters of each experiment are orthogonal [38]. Table 5 shows the main effects of the alignment parameters and the filters. Each main effect was calculated using the average of a design factor at a given level. As an example, level 2 of the alignment parameter, $l$, is in the $2^{\text {nd }}, 4^{\text {th }}$ and $9^{\text {th }}$ experiments and their corresponding image quality scores are $16.218,17.080$ and 16.931 respectively. The main effect of the alignment parameter, $l$, with level 2 is the average of the image quality scores of these experiments which is given by 16.743 (i.e. $(16.218+17.080+16.931) / 3)$.

Table 5 Main effects of the Design Factors.

\begin{tabular}{|c|c|c|c|c|}
\hline \multirow{2}{*}{ Parameter levels } & \multicolumn{3}{|c|}{ Alignment parameters } & \multirow{2}{*}{ Filter } \\
\cline { 2 - 4 } & $a$ & $b$ & $l$ & \\
\hline Level 1 & $\underline{\mathbf{1 6 . 3 8 2}}$ & 17.443 & 17.368 & 16.969 \\
\hline Level 2 & 16.752 & 16.731 & 16.743 & 16.89 \\
\hline Level 3 & 17.394 & $\underline{\mathbf{1 6 . 3 5 4}}$ & $\underline{\mathbf{1 6 . 4 1 7}}$ & $\underline{\mathbf{1 6 . 6 7}}$ \\
\hline Sensitivity & 1.0113 & 1.089 & 0.95033 & 0.299 \\
\hline
\end{tabular}

The smallest main effect of each alignment parameter are highlighted in bold and underlined in Table 5, i.e. $a$ with level 1 (or $a=-1.5$ ), $b$ with level 3 (or $b=1.5$ ), $l$ with level 3 (or $l=4.9$ ) and the filter with level 3 (or Regularized filter) is same than that obtained by the $3^{\text {rd }}$ experiment on $L_{9}(3)$. Hence, the appropriate setting is given by the $3^{\text {rd }}$ experiment on $L_{9}$ (3) i.e.: $a$ with level 1 (or $a=-1.5$ ), $b$ with level 3 (or $b=1.5$ ), $l$ with level 3 (or 
$l=4.9$ ), and the filter with level 3 (or Regularized filter). With the setting of the $3^{\text {st }}$ experiment, the image score is given as 15.295 . Slightly better image quality is obtained with $L_{9}(3)$ than that obtained based on the main effects. Hence, nonlinearity between the alignment parameters and the filter is not high. Although further calibration can be conducted by dividing the design factors with more levels, only a much smaller improvement can be expected, as it is found that a small difference exists between the result obtained based on the main effects and that obtained by the experiments of the $L_{9}(3)$. Therefore, we terminate the calibration and use the determined levels of design factors obtained based on $L_{9}(3)$ as the calibration result.

The sensitivity of each design factor is computed by taking the difference between the largest and smallest main effect for a given design factor. It reveals that the alignment parameter, $a$, shows the greatest sensitivity. Hence, the alignment parameter, $a$, has the greatest effect on the image quality by varying its value. The other Design Factors (i.e. alignment parameters $b$ and $l$, and the filter) has the least sensitivity to the image quality. Hence, there is little effect on the image quality when we tune these design factors. The main effects of the design factors are also shown graphically in Figure 2. Graphing the main effects of all design factors can provide more insight at a glance, and it clearly shows that the alignment parameter, $a$, has much greater sensitivity than those of the other design factors.

Figure 3 depicts the original image and Figure 4 depicts the deblurred image generated using the calibrated alignment parameters and the selected filter. The deblurred image shows that the blurred effect is removed from the original image and the object’s edges are clearer. 

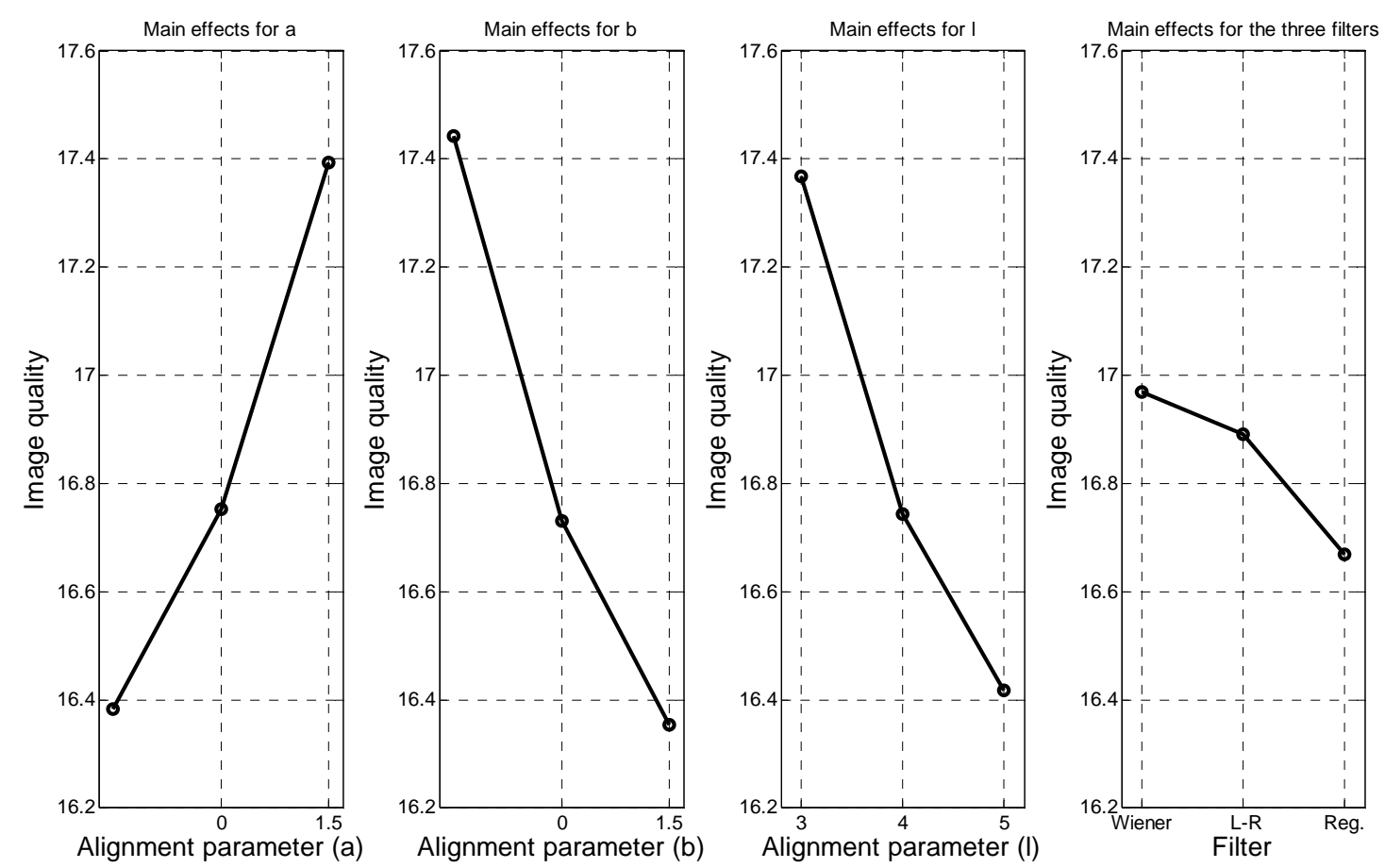

Figure 2 Illustration of the main effects of the Design Factors.

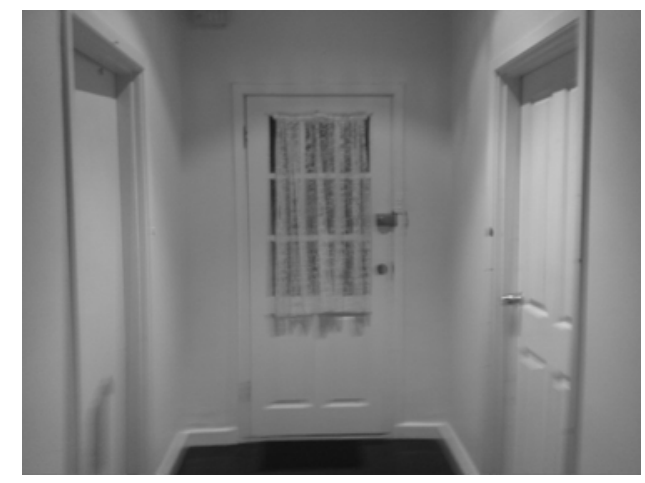

Figure 3 Original image.

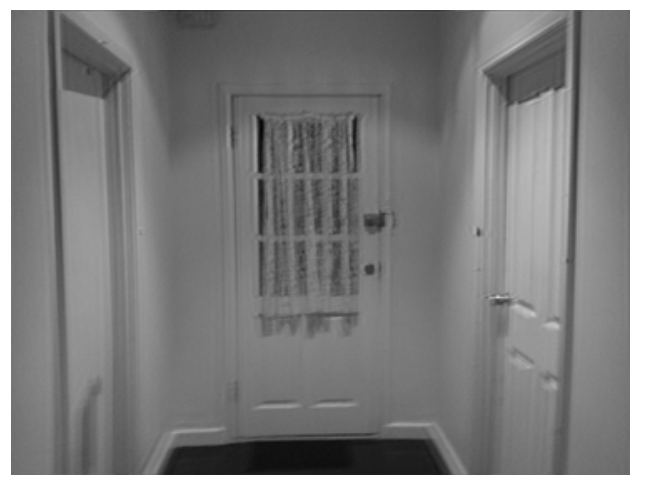

Figure 4 Deblurred image.

\section{RESULT EVALUATION}

The result obtained by the Taguchi method was evaluated through two phases. In the first phase, the obtained result was evaluated with those obtained by the commonly-used deblurring methods, where the alignment parameters defaulted by the camera manufacturer were engaged with those deblurring methods. The deblurring performance was evaluated by several deblurring metrics. We attempt to evaluate whether 
significantly better results can be obtained by the Taguchi method than the commonly-used deblurring methods engaged with the defaulted parameters. In the second phase, several commonly-used optimization methods were used to determine the alignment parameters and the deblurring filter. We attempt to evaluate whether the Taguchi method can obtain better results than those optimization methods, when similar computational time is used.

\subsection{Comparison with defaulted alignment parameters}

Four commonly used deblurring methods [12] namely Blind Deconvolution filter, Wiener filter, Lucy-Richardson filter, and regularized filter, were used to deblur the original image. The alignment parameters defaulted by the camera manufacturer were used to develop the blur kernel, where $a=0, b=0$, and $l=4$. Apart from Mittal et al's image quality measure, three commonly used metrics namely no-reference blur [21], gradients based measure [33] and edges based measure [33] were used to evaluate the effectiveness of the deblurring filters.

Figure 5a shows the original image score, and the deblurred image scores obtained by the four deblurring methods and the proposed Taguchi method. The image quality is better when the degree of Mittal et al's score is smaller. It shows that the score obtained by the proposed Taguchi method is the best comparing to the original image and the other tested deblurring methods. To further illustrate the performance of the proposed Taguchi method, Figure 5b shows the relative improvements when each of the tested methods is compared with the proposed method. The relative improvement is the difference between the scores obtained by the proposed method and the each of the other tested method. The proposed method obtained the improvement with more than $20 \%$ relatively to the original image, and also it obtained the improvements with more than $8 \%$ relatively to the other tested methods. 


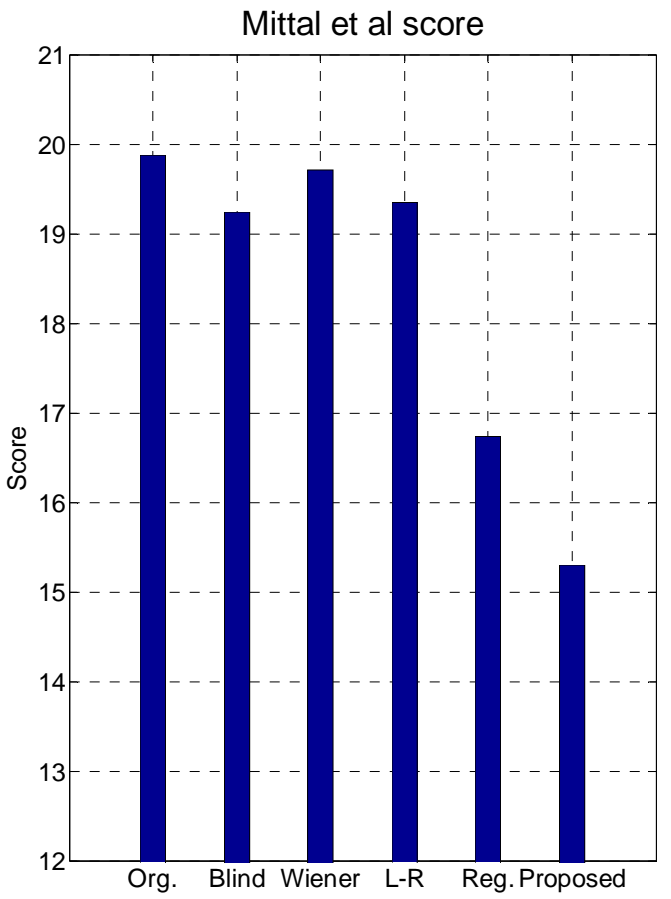

Figure 5a Mittel et al’s score

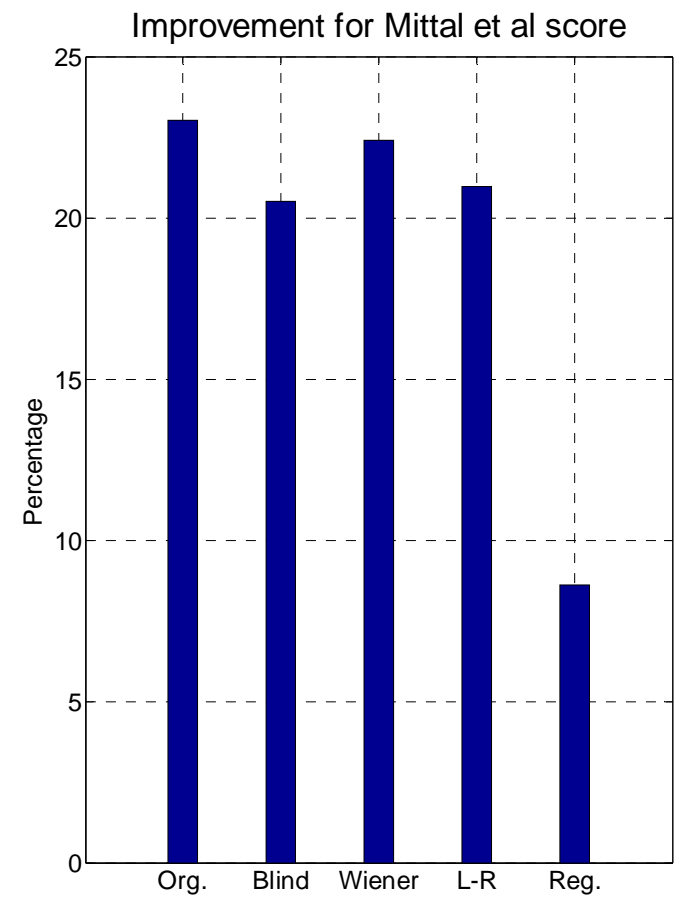

Figure 5b Improvement of Mittal et al's score

A supplemental metric, no-reference blur [21], was used to evaluate the amount of blur in the original image and the images obtained by the five deblurring methods. It accounts the smoothing effect of blur by measuring the distance between edges on the image. For pixels that correspond to an edge point, the local extrema in the corresponding image are used to compute the edge width. The edge width then defines a local measure of blur. The global blur measure is determined based on the average of the local blur values over all edge locations. Therefore this metric accounts in-block blur but rather contributes a global blur measure. Figure 6a shows the blur degrees of the original image and the images obtained by the deblurring methods. It shows that blur degree obtained by the proposed Taguchi method is the smallest compared with the other tested methods and the original image. Figure $6 \mathrm{~b}$ also shows that improvements with more than $20 \%$ can be obtained by the proposed Taguchi method relatively to the original image, blind deconvolution filter, Wiener filter, and Lucy-Richardson method. Also an improvement with about $4 \%$ can be obtained relatively to the regularized filter. 


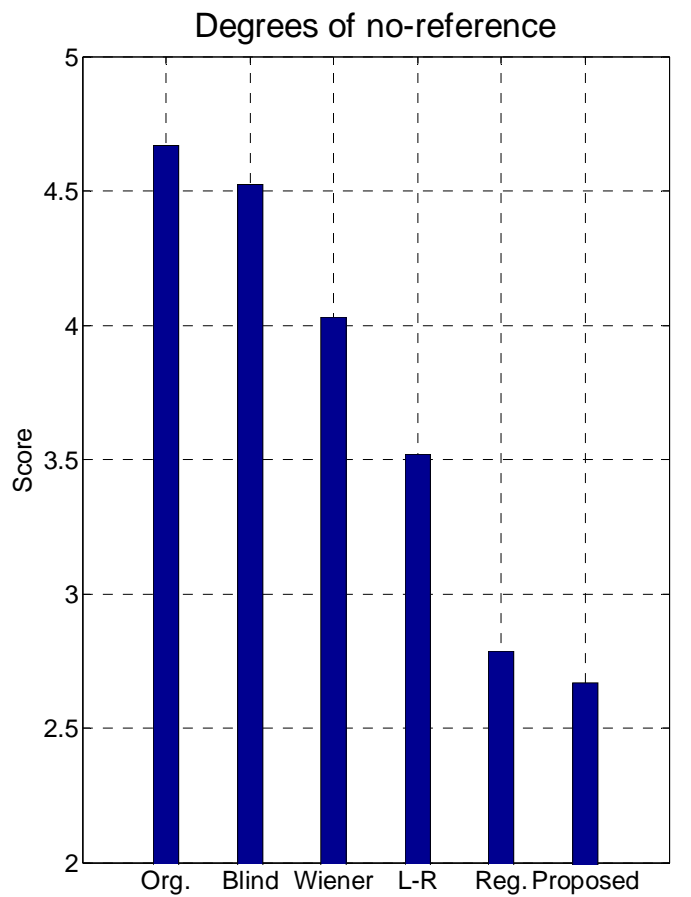

Figure 6a no-reference measure

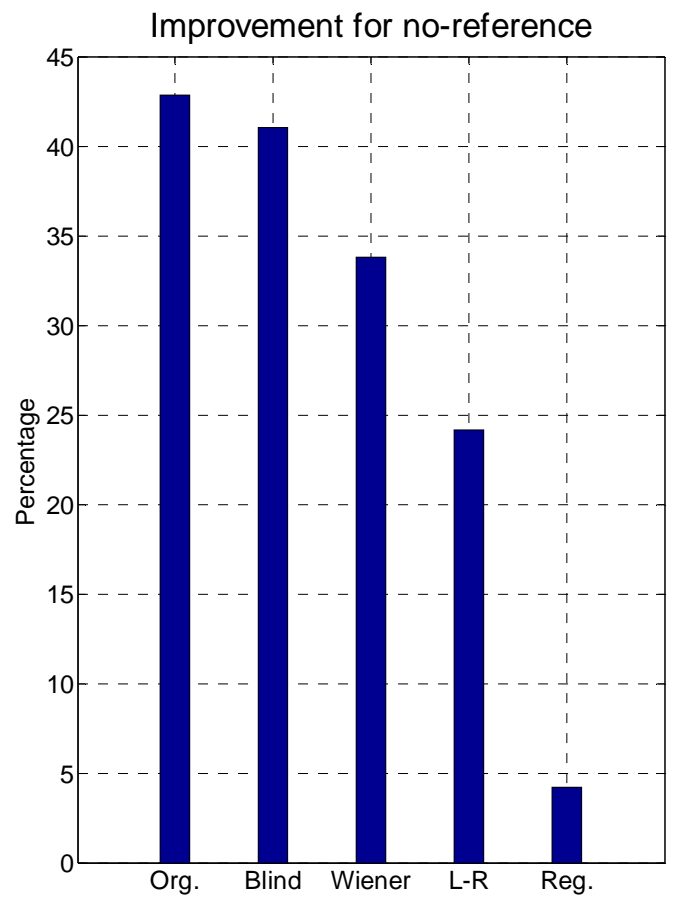

Figure 6b Improvement of no-reference measure

Also, two image activity measures based on gradients and edges [33] were used to quantify the amount of blur on the images. These measures have already been successfully used to measure blur artifacts without reference [41]. These measures relate to the amount of local changes in the images caused by strong local contrasts and noise. The more changes, the greater is the activity. Blur typically reduces the amount of image activity as the image is smoothed and has lesser changes. Hence, more blur exists on the image, when smaller activities are detected. The measures based on gradients and edges are shown in Figures 7 and 8 respectively. They shows the image activity obtained by the proposed Taguchi method is the highest compared with the original image and the other tested methods. As the blur has the opposite effect to the amount of image activity, the two activity measurements are in full agreement with visual inspection which is the highest for the deblurred image obtained by the Taguchi method. They further validate the effectiveness of the deblurring, when the Taguchi method is used to determine the alignment parameters. Therefore, the measures for these four image quality metrics show that better image quality can be obtained by the proposed Taguchi method than the other deblurring filters which is engaged with the defaulted alignment parameters. 


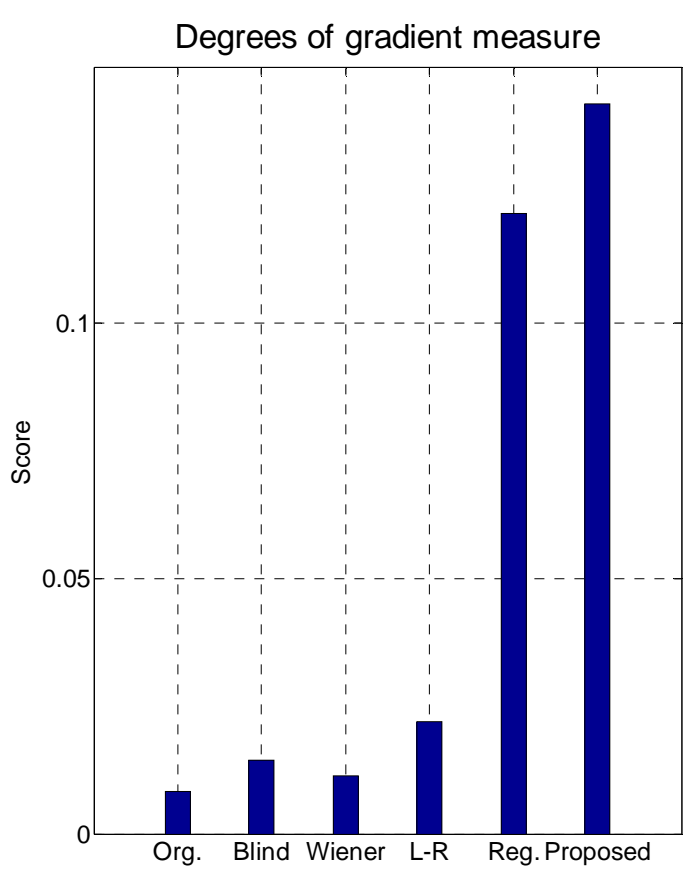

Figure 7a Degrees of gradient measure

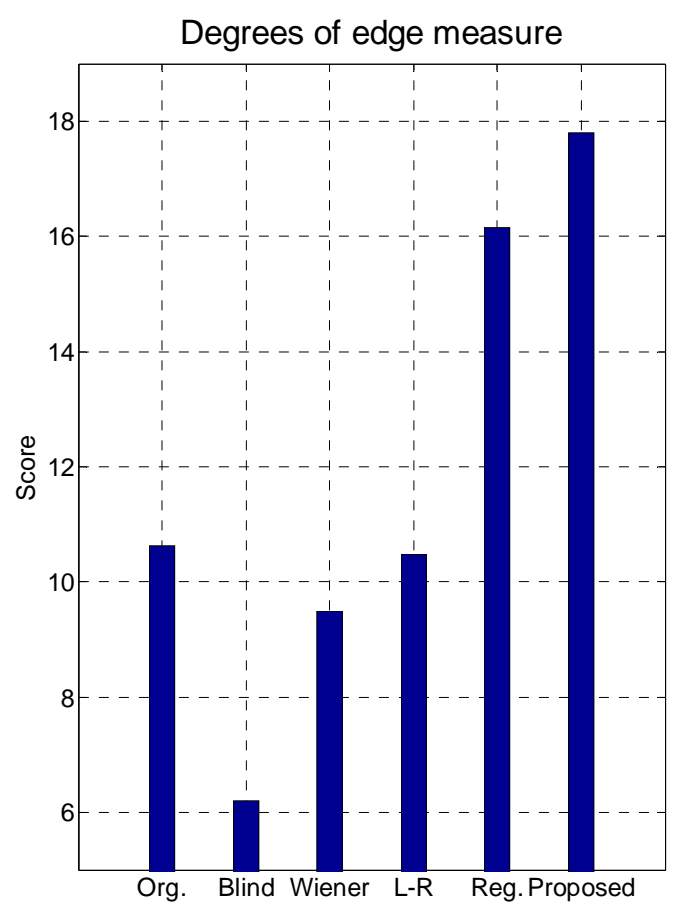

Figure 8a Degrees of edge measure

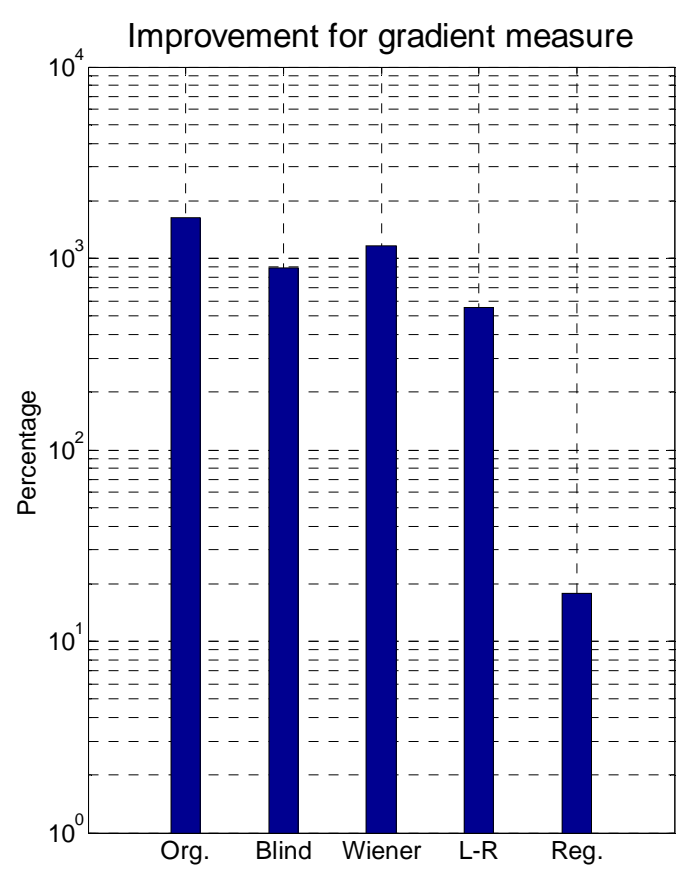

Figure 7b Improvement of gradient measure

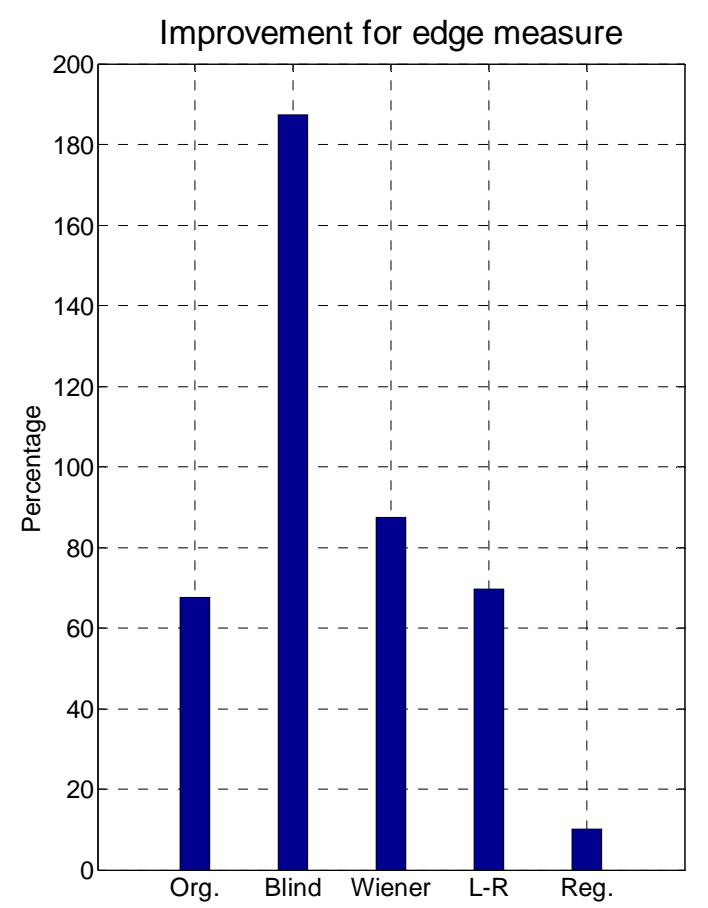

Figure 8b Improvement of edge measure 


\subsection{Comparison with commonly-used optimization methods}

Several commonly-used methods including genetic algorithm (GA) [10], particle swarm optimization algorithm (PSO) [28] and simulated annealing (SA) [16] have been applied to determine the four appropriate design factors (i.e. the three alignment parameters and the deblurring filter). These optimization methods were tested, as they are usually applied to solve difficult optimization problems and have been used on solving many instrument design problems [1, 6, 18, 26, 31, 48]. Based on these comparisons, the effective of the proposed Taguchi method can be further evaluated. The following parameters and operations were used in the three heuristic methods:

1. Genetic algorithm (GA): Two genetic algorithms namely GA-3-3 and GA-3-9 have been used to determine the four Design Factors of the calibration problem, where GA-3-3 involves 3 chromosomes and 3 generations and GA-3-9 involves 3 chromosomes and 9 generations. Hence, the same numbers of computational evaluations are used for both the GA-3-3 and the Taguchi method, and three times the number of computational evaluations are used for the GA-3-9 than those used for the Taguchi method.

In both GA-3-3 and GA-3-9, the chromosomes are coded as parametrical representations with four genes, the first three of which represent the three alignment parameters (i.e. $a, b$, and $l$ ). The fourth gene represents the three filters (i.e. Wiener filter, Lucy-Richardson filter and regularized filter) whereby the gene is divided into three domains. The first domain represents the Wiener filter. The second and third domains represent the Lucy-Richardson filter and regularized filter respectively. In the two GAs, each chromosome is evaluated based on the image quality analyzer [23] which is used here in the Taguchi method. The detailed evolutionary operations can be found in [25]. The following parameters were used in both GA-3-3 and GA-3-9: crossover rate $=0.8$; and mutation rate $=0.1$.

2.Particle swarm optimization (PSO): The two particle swarm optimization algorithms, namely PSO-3-3 and PSO-3-9, have been used to determine the four design factors of the calibration problem. The PSO-3-3 involves 3 particles and 3 iterations and PSO-3-9 involves 3 particles and 9 iterations. 
Hence, the same numbers of computational evaluations are used for both PSO-3-3 and the Taguchi method. This allows us to determine whether the PSO algorithm can outperform the Taguchi method, when the same computational effort is involved. PSO-3-9 required three times the computational evaluations compared with the Taguchi method. This can determine whether the Taguchi method can still outperform the PSO algorithm when much more computational effort is used in the PSO.

The particle representation of both PSO-3-3 and PSO-3-9 is same as that coded in the two GAs. In the two PSO algorithms, each particle is evaluated based on the image quality analyzer [23]. The following PSO parameters were used: the maximum and minimum inertia weights are set to 0.9 and 0.2 , respectively; the initial acceleration coefficients are set to 2.0.

3. Simulated annealing (SA) algorithm: The two simulated annealing methods [16] namely SA-9 and SA-27 have been used to determine the four Design Factors of the calibration problem. The SA-9 and the SA-27 were involved with 9 and 27 iterations respectively, where SA-9 needed the same computational effort as the Taguchi method and SA-27 needed three times the computational effort of the Taguchi method. Both methods attempted to evaluate whether Taguchi method can outperform the SA algorithms when same and more computational efforts were used. In the two SA algorithms, each iterative solution is evaluated based on the image quality analyzer [23].

All these methods were implemented using Matlab 7.7 in a PC which has a CPU of Intel(R) Core(TM) 2 Duo $2.66 \mathrm{GHz}$ and a memory of 8GB. As the GA, PSO and SA, are heuristic methods, different results are generated under different runs. Therefore, all the tested methods, GA-3-3, GA-3-9, PSO-3-3, PSO-3-9, SA-1-9 and SA-1-27, were run 30 times, and the results of the 30 runs were recorded. The computational evaluations and computational time used in all the tested methods are given in Table 6. Results in terms of image quality scores obtained by all the tested methods are shown in the boxplot given in Figure 9. It shows that the image quality obtained by GA-3-3, PSO-3-3 and SA-1-9 are worse than those for the Taguchi method, where the computational evaluations used by the four methods were the same. Figure 9 also shows that the image quality obtained by the Taguchi method are slightly worse than those for GA-3-9, PSO-3-9, 
and SA-1-27, while the computational evaluations used by the GA-3-9, PSO-3-9, and SA-1-27 were three times more than those used by the Taguchi method. Also, the heuristic methods, GA-3-9, PSO-3-9 and SA-1-27, used more than three times the computational time than that used by the Taguchi method, as the two methods required more computational operations than those required by the Taguchi method. Therefore, much more computational time and effects are required by GA-3-9, PSO-3-9 and SA-1-27 in comparison with the Taguchi method, although slightly better image quality can be obtained by the two methods.

Table 6 Computational evaluations and computational time used on the 7 tested methods.

\begin{tabular}{|c|c|c|}
\hline Methods & Computational evaluations & Computational time (seconds) \\
\hline Original image & N/A & 174.03 \\
\hline GA-3-3 & 9 & 167.03 \\
\hline PSO-3-3 & 9 & 165.54 \\
\hline SA-1-9 & 9 & 500.72 \\
\hline GA-3-9 & 27 & 491.47 \\
\hline PSO-3-9 & 27 & 487.68 \\
\hline SA-1-27 & 27 & 163.75 \\
\hline Taguchi method & 9 & \\
\hline
\end{tabular}




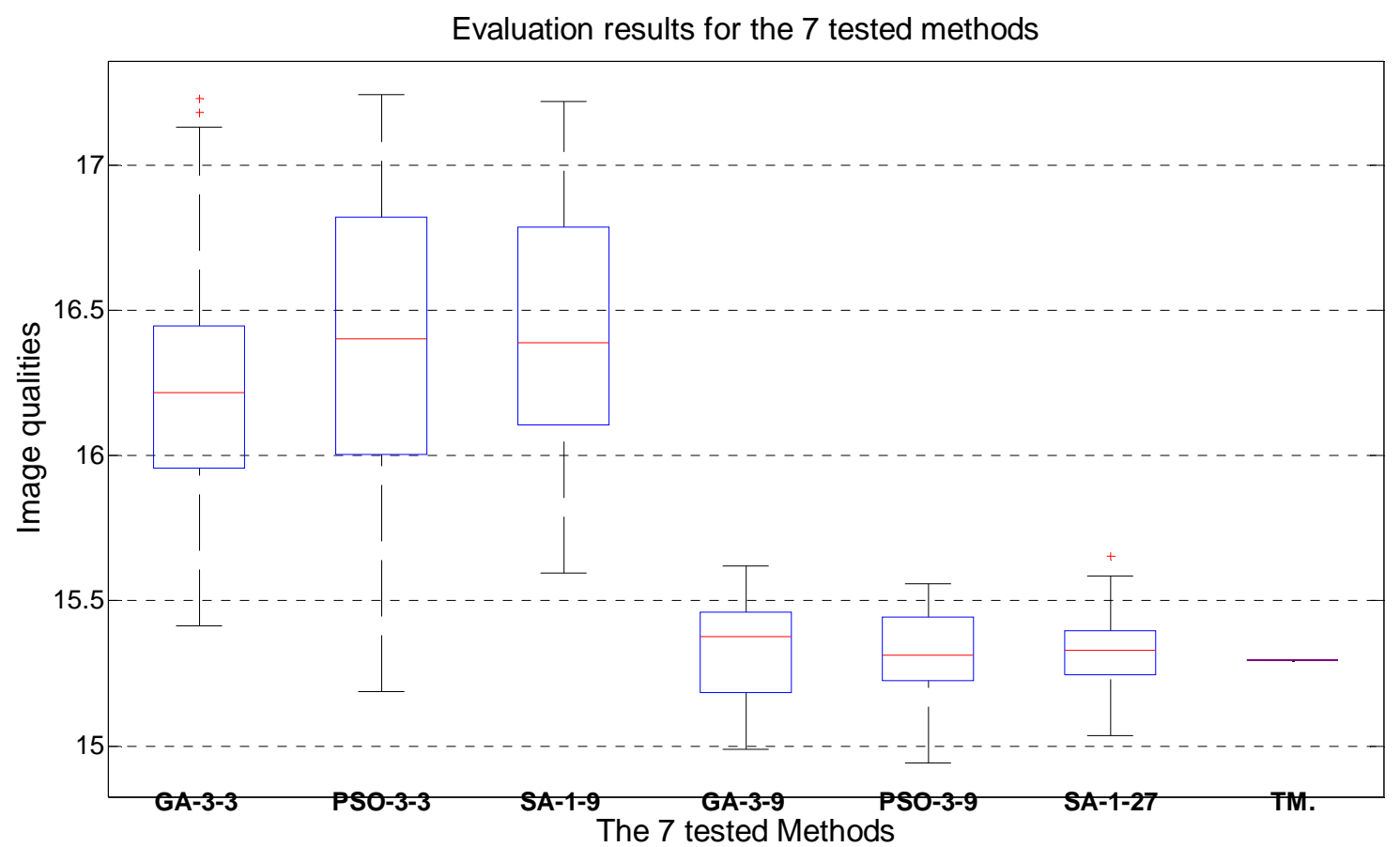

Figure 9 Evaluation results for the tested optimization methods.

Table 7 shows the averages of the image quality scores for the 30 runs of the tested methods. It shows that better image quality can be obtained by the Taguchi method which achieves an improvement of $20 \%$ compared with the original image based on the image quality analyzer [23]. It also shows that the average image quality scores obtained by all the tested methods are very similar. It is difficult to claim the significance of the image quality differences between the Taguchi method and the other tested methods (i.e. GA-3-3, GA-3-9, PSO-3-3, PSO-3-9, SA-1-9 and SA-1-27), although we can see that the image quality obtained by the Taguchi method is better than those obtained by GA-3-3, PSO-3-3 and SA-1-9 which require the same computational efforts as the Taguchi method. Therefore, the $t$-test [5] was used to evaluate the significance of the hypothesis that the sample means of the image quality scores obtained by the proposed Taguchi method are better than those obtained by the other tested methods. Based on the $t$-distribution table, if the $t$-value is higher than 1.96, the image quality obtained by the Taguchi method is better than those of the other methods at a $97.5 \%$ confidence level. 
The $t$-value for statistical significance testing between the Taguchi method and any of the other methods is given by:

$$
t \text {-value }=\frac{\mu_{2}-\mu_{1}}{\sqrt{\sigma_{2}^{2} / N_{2}+\sigma_{1}^{2} / N_{1}}},
$$

where $\mu_{1}$ and $\mu_{2}$ denote the respective mean image quality, $\sigma_{1}^{2}$ and $\sigma_{2}^{2}$ the image quality variance, and $N_{1}$ and $N_{2}$ the number of tests. Here both $N_{1}$ and $N_{2}$ are equal to 30. As the Taguchi Method is a deterministic method, an identical solution can be obtained with different runs. Hence, the variance for the Taguchi method is zero.

For example, we consider the $t$-value between Taguchi method to GA-3-3 is calculated as

$$
t \text {-value }=\frac{16.440-15.295}{\sqrt{0.172 / 30+0 / 30}}=15.122
$$

where the mean image quality obtained by GA-3-3 is 16.440 ; the mean image quality obtained by the Taguchi method is 15.295 ; the variance of the image quality obtained by GA-3-3 is 0.172 ; as mentioned, the variance obtained by the Taguchi method is 0 since the Taguchi method is the deterministic method.

If the $t$-value is higher than 1.699, the significance is $95 \%$ confidence level, which means that the image quality obtained by the Taguchi method are different with those obtained by the GA-3-3 with 95\% confidence level. In general, the results indicate that $t$-values between the Taguchi method to the GA-3-3, PSO-3-3 and SA-1-9 are greater than 1.96, where these methods involved the same computation evaluations as the Taguchi method. This means the Taguchi Method can obtain difference image qualities with 95\% confidence level compared with those generated by the three tested methods, GA-3-3, PSO-3-3 and SA-1-9. The image quality is better, when the small image quality is obtained. Hence, the image quality obtained by the Taguchi method is significantly better with 95\% conference level than those obtained by GA-3-3, PSO-3-3 and SA-1-9. Although the other three tested methods, GA-3-9, PSO-3-9 and SA-1-27, used more than three times the computational effort that was required by the Taguchi method, these three tested methods cannot achieve image quality difference with 95\% confidence level compared with the Taguchi method, as the absolute $t$-values achieved by these three methods are less than the 1.96. Therefore, although GA-3-9, PSO-3-9 and 
SA-1-27, can obtained better image qualities than those obtained by the Taguchi method, there is no significant difference between the image quality obtained between either GA-3-9, PSO-3-9 or SA-1-27 to the Taguchi method. Also based on Table 6, much longer computational time was required by GA-3-9, PSO-3-9 and SA-1-27 compared with the Taguchi method. Based on the $t$-test, the effectiveness of the Taguchi method can be further demonstrated based on the $t$-tests.

Table 7 Averages of image quality scores obtained by the 7 tested methods.

\begin{tabular}{|c|c|c|c|}
\hline \multirow{2}{*}{ Methods } & \multicolumn{3}{|c|}{ Image quality scores } \\
\cline { 2 - 4 } & Means & Variances & t-test \\
\hline Original image & 19.878 & 0 & N/A \\
\hline GA-3-3 & 16.440 & 0.172 & 15.122 \\
\hline PSO-3-3 & 16.381 & 0.296 & 10.933 \\
\hline SA-1-9 & 16.287 & 0.276 & 31.906 \\
\hline GA-3-9 & 15.331 & 0.029 & 1.158 \\
\hline PSO-3-9 & 15.253 & 0.028 & -1.351 \\
\hline SA-1-27 & 15.264 & 0.020 & -1.201 \\
\hline Taguchi Method & 15.295 & 0 & N/A \\
\hline
\end{tabular}

\section{CONCLUSIONS}

This paper proposed the Taguchi method as a means of calibrating the alignment parameters for IMUs and determining the appropriate filter for image deblurring. The proposed Taguchi method first conducts a small but systematic number of experiments based on the orthogonal array, and then it studies the impact of each alignment parameter and the deblurring filter, in order to determine the appropriate levels for the alignment parameters and the deblurring filter. Several widely adopted image quality metrics were used to evaluate the deblurred images generated by the proposed Taguchi method. The results show that the quality of deblurred images achieved by the proposed Taguchi method is better than that obtained by deblurring method, which 
has not addressed the alignment parameter calibration and filter selection. The results were also compared with those obtained by the commonly-used heuristic methods which are effective in solving hard optimization problems with no gradient information. The results show that the Taguchi method obtains better image quality than the tested optimization methods, while maintaining similar computational complexity..

This paper has demonstrated how the alignment parameters can be calibrated based on the Taguchi method. In future work, the Taguchi method can be investigated by incorporating with the IMU built-in functionality in order to calibrate the alignment parameters automatically. To do this, the Taguchi method and the image quality metric will be embedded on the smartphone processor. Apart from evaluating the image quality, computational time used on the smartphone processor will be measured.

\section{REFERENCES}

[1] V. Akbarzadeh, C. Gagne, M. Parizeau, M. Argany and M.A. Mostafavi, Probabilistic Sensing Model for Sensor Placement Optimization Based on Line-of-Sight Coverage, IEEE Transactions on Instrumentation and Measurement 62(2) (2013) 293-303.

[2] I. Asilturk and S. Neseli, Multi response optimization of CNC turning parameters via Taguchi method based response surface analysis, Measurement 45 (2013) 785-794.

[3] H. Bae and C. Fowlkes, Accurate motion deblurring using camera motion tracking and scene depth, Proceedings of IEEE Workshop on Applied Computer Vision (2013).

[4] T. Beravs, J. Podobnik and M. Munih, Three axial accelerometer calibration using Kalman filter covariance matrix for online estimation of optimal sensor orientation, IEEE Transactions on Instrumentation and Measurement 61 (9) (2012): 2501-2511.

[5] G. E.-P. Box, J. S. Hunter, and W. G. Hunter, Statistics for Experiments: Design, Innovation, and Discovery, 2nd ed. New York: Wiley 2005.

[6] M.Z. Daud, A. Mohamed, A.A. Ibrahim and M.A. Hannan, Heuristic optimization of state-of-charge feedback controller parameters for output power dispatch of hybrid photovoltaic/battery energy storage system, Measurement 49 (2014) 15-25. 
[7] O. Erkan, M. Demetgul, B. Isik and I.N. Tansel, Selection of optimal machining conditions for the composite materials by using Taguchi and GONNs, Measurement 48 (2014) 306-313.

[8] R. Fergus, B. Singh, A. Hertzmann, S. T. Roweis, and W. Freeman, Removing camera shake from a single photograph, ACM Transactions on Graphics 25 (2006) 787-794.

[9] J. Rivera and R.V.D. Meulen, Gartner says annual smartphones sales surpassed sales of feature phones for the first time in 2013, Gartner (2013).

[10] D.E. Goldberg, Genetic Algorithms in Search, Optimization and Machine Learning, Boston, MA, USA: Addision Wesley Longman Publishing Co., Inc., (1989).

[11] B. Gunter, A perspective on the Taguchi methods, Quality Progress (1987) 44-52.

[12] R. Horstmeyer, Camera motion tracking for deblurring and identification, Technical Report, MIT Media Laboratory, MIT (2010).

[13] A.K. Jain, Ed., Fundamentals of Digital Image Processing, New York, USA: Prentice Hall (1989).

[14] X. Jiang, D.C. Cheng, S. Wachenfeld and K. Rothaus, Motion deblurring, Technical Report, Department of Mathematics and Computer Science, University of Muenster (2004).

[15] N. Joshi, S.B. Kang, and C.L. Zitnick, Image deblurring using inertial measurement sensors, ACM Trans. Graphics 29 (4) (2010) 1-9.

[16] S. Kirkpatrick, C. D. G. Gelatt, Jr., andM. P. Vecchi, “Optimization by simulated annealing”, Science 220 (1983) 671-680.

[17] C.K. Kwong, K.Y. Chan and H. Wong, An empirical approach to modeling fluid dispensing for electronic packaging, International Journal of Advanced Manufacturing Technology 34 (1-2) (2007) $111-121$.

[18] C.C. Lai and Y.C. Chen, A user-oriented image retrieval system based on interactive genetic algorithm, IEEE Transactions on Instrumentation and measurement 60 (10) (2011) 3318-3325.

[19] N.D. Lane, E. Miluzzo, H. Lu, and D. Peebles, A survey of mobile phone sensing, IEEE Communications Magazine 48 (9) (2010) 140-150.

[20] J. Li, J. Fang and M. Du, Error analysis and gyro-bias calibration of analytic coarse alignment for 
airborne POS, IEEE Transactions on Instrumentation and Measurement 61 (11) (2012) 3058-3064.

[21] P. Marziliano, F. Dufaux, S. Winkler, T. Ebrahimi, A no-reference perceptual blur metric, in Proceedings of IEEE International Conference Image Processing (2002) 57-60.

[22] R.J. Mayer and P.C. Benjamin, Using the Taguchi paradigm for manufacturing system design using simulation experiments, Industrial Engineering, 22 (2) (1992) 195-209.

[23] A. Mittal, R. Soundararajan, and A.C. Bovik, Making a Completely Blind Image Quality Analyzer, IEEE Signal Processing Letters, 20 (2013) 209-212.

[24] A. K. Moorthy and A. C. Bovik, Blind image quality assessment: From natural scene statistics to perceptual quality, IEEE Transactions Image Processing, 20 (12) (2011) 3350-3364.

[25] H. Muhlenbein and D.S. Voosen, Predictive models for the breeder genetic algorithm: I. Continuous parameter optimization, Evolutionary Computation 1 (1) (1993) 25-49.

[26] H.A. Nguyen, H. Guo and K.S. Low, Real-time estimation of sensor node's position using particle swarm optimization with log-barrier constraint, IEEE Transactions on Instrumentation and Measurement 60 (11) (2011) 3619-2628.

[27] M. Park and Y. Gao, Error and performance analysis of MEMS-based inertial sensors with a low-cost GPS receiver, Sensors, 8 (2008) 2240-2261.

[28] K.E. Parsopoulos and M.N. Vrahatis, On the computation of all global minimizers through particle swarm optimization, IEEE Transactions on Evolutionary Computation, 8 (3) (2004) 211-224.

[29] J. Prasanna, L. Karunamoorthy, M.V. Raman, S. Prashanth and D.R. Chordia, Optimization of process parameters of small hole dry drilling in Ti-6Al-4V using Taguchi and grey relational analysis, Measurement 48 (2014) 364-354.

[30] A. Rav-Acha and S. Peleg, Two motion blurred images are better than one, Pattern Recognition Letters 26 (2005) 311-317.

[31] N.K. Rout, D.P. Das and G. Panda, Particle swarm optimization based active noise control algorithm without secondary path identification, IEEE Transactions on Instrumentation and Measurement 62 (2) (2012) 554-562. 
[32] M. Saad, A. C. Bovik, and C. Charrier, Blind image quality assessment: A natural scene statistics approach in the DCT domain, IEEE Transactions Image Processing 21 (8) (2012) 3339-3352.

[33] S. Saha, R. Vemuri, An analysis on the effect of image features on lossy coding performance, IEEE Signal Processing Letters 7 (5) (2000) 104-107.

[34] D.P. Selvaraj, P. Chandramohan and M. Monanraj, Optimization of surface roughness, cutting force and tool wear of nitrogen alloyed duplex stainless steel in dry turning process using Taguchi method, Measurement 49 (2014) 205-215.

[35] Q. Shan, J. Jia and A. Agarwala, High-quality motion de-blurring from a single image, in Proceedings of ACM SIGGRAPH Conference (2008).

[36] O. Sindelar and F. Sroubek, Image de-blurring in smartphone devices using built in inertial measurement sensors, Journal of Electronic Imaging 22 (2013) 1-22.

[37] L.P. Sullivan, The power of Taguchi methods, Quality Progress (1987) 76-79.

[38] G. Taguchi, Quality engineering (Taguchi methods) for the development of electronic circuit technology, IEEE Transactions on Reliability 44 (2) (1995) 225-229.

[39] G. Taguchi, Y. Yokohama, and Y. Wu, Eds., Design of experiments. Tokyo, Japan: ASI Press, (1993).

[40] D. Titterton and J.L. Weston, Eds., Strapdown Inertial Navigation Technology. IET Publisher, $2^{\text {nd }}$ Edition, (2004).

[41] E. Ulrich, K. Maulana, H.J. Zepernick and M. Caldera, Reduced reference matric design for objective perceptual quality assessment in wireless imaging, Signal Processing, 24 (7) (2009) 525-547.

[42] Z. Wang, A. C. Bovik, H. R. Sheikh, and E. P. Simoncelli, Image quality assessment: From error visibility to structural similarity, IEEE Transactions on Image Processing, 13 (4) (2004) 600-612.

[43] C. Wang, Y. Yue, F. Dong, Y. Tao, X. Ma, G. Clapworthy and X. Ye, Enhancing Bayesian estimators for removing camera shake, Computer Graphics Forum, vol. 32, no. 6, pp. 113-125, 2013.

[44] C. Wang, Y. Yue, F. Dong, Y. Tao, X. Ma, G. Clapworthy, H. Lin and X. Ye, Nonedge specific adaptive scheme for highly robust blind motion deblurring of natural images, IEEE Transactions on Image Processing, vol. 22, no. 3, pp. 884-897, 2013. 
[45] P. Ye and D. Doermann, No-reference image quality assessment using visual codebook, in Proceedings of IEEE International Conference Image Processing (2011).

[46] L. Yuan, J. Sun, L. Quan, and H.Y. Shum, Image de-blurring with blurred/noisy image pairs, in Proceedings of ACM SIGGRAPH Conference (2007).

[47] X. Yun, J. Calusdian, E.R. Bachmann and R. McGhee, Estimation of human foot motion during normal walking using inertial and magnetic sensor measurements, IEEE Transactions on Instrumentation and Measurement 61 (7) (2012) 2059-2072.

[48] K. Zhu, X. Song and D. Xue, A roller bearing fault diagnosis method based on hierarchical entropy and support vector machine with particle swarm optimization algorithm, Measurement, 47 (2014) 669-675. 